\title{
Just the facts: Hypertonic saline is just as good as (and probably better than) mannitol
}

\author{
Laurel Murphy, MD, FRCPC*
}

CLINICAL SCENARIO

A 27-year-old female is brought to the emergency department (ED) by ambulance following a motor vehicle collision at highway speed. She was the belted driver. She has no significant past medical history and is on no medications. Following a prolonged extrication, she is intubated due to decreased level of consciousness before transport.

On arrival to the ED, she is afebrile, with a blood pressure of 97/41, a heart rate of 113 and an oxygen saturation of $100 \%$ on an $\mathrm{FiO}_{2}$ of 1.0 on the ventilator. Her initial Glasgow coma scale (GCS) score is E1VTM3 (4T) on no sedation, and her pupils are $3 \mathrm{~mm}$ and reactive. She has bilateral open femur fractures. While you are applying splints to her fractures before sending her for computed tomography (CT) scan, the RN reports that her right pupil is now $8 \mathrm{~mm}$ and nonreactive.

\section{KEY CLINICAL QUESTIONS}

\section{What are the initial steps in management when raised intracranial pressure is suspected?}

In Emergency Medicine, we commonly have to make treatment decisions for our patients before establishing a diagnosis. The case above describes a patient who very likely has raised intracranial pressure from hemorrhage or cerebral edema related to her trauma. The deterioration in her neurological status from a reactive to a now dilated, nonreactive pupil is an indication for immediate treatment, despite not having yet obtained the CT scan.

To manage this patient, we should first use the usual noninvasive measures for raised intracranial pressure including elevating the head of the bed, ensuring there is no venous obstruction of the neck veins including a tight $\mathrm{C}$-spine collar, increasing sedation, and paralyzing the patient. In addition, ensure normal oxygenation, normocarbia and normal blood glucose levels. If there is no improvement following these, a hyperosmotic agent can be given empirically. The two options are mannitol (0.25-1 g/ kg over 20-30 minutes) and hypertonic saline (150mL bolus of 3\%). It is important to be aware of the concentration of hypertonic saline available at your center, as the doses are significantly different (Table 1). Hypertonic saline would be a very reasonable choice for this patient.

Table 1. Dosing of HTS by concentration

From the * Dalhousie University, QEll Health Sciences Center, Departments of Emergency Medicine and Critical Care, Halifax, NS.

Correspondence to: Dr. Laurel Murphy, Dalhousie University, QEII Health Sciences Center, Departments of Emergency Medicine and Critical Care, Room 348, Bethune Building, 1276 South Park Street, Halifax, NS B3H 2Y9; Email: laurelmurphy@gmail.com

(c) Canadian Association of Emergency Physicians

CJEM 2020;22(2):152-154

DOI 10.1017/cem.2019.455 


\section{How do hyperosmotic agents work to reduce intracranial pressure?}

The human brain is made up of approximately $80 \%$ water. This means that we can alter the volume of brain tissue by influencing the movement of water across the blood-brain barrier. Hyperosmotic agents are impermeable to the bloodbrain barrier, and, thus, create an osmotic gradient that causes water to move from the brain across the blood-brain barrier to the area of higher concentration in the serum. This exodus of water results in a decrease in brain tissue volume and, as a result, intracranial pressure.

\section{When should I use a hyperosmotic agent to treat raised intracranial pressure?}

In a patient with a history consistent with raised intracranial pressure, hyperosmotic agents should be used when there are signs of impending herniation despite noninvasive measures and high doses of sedation \pm paralysis. The following are reasonable clinical indications to treat with a hyperosmotic agent: coma, worsening neurologic function, anisocoria (pupils unequal size), decorticate or decerebrate posturing, hypertension and bradycardia (Cushing reflex).

\section{What are the advantages and disadvantages of hypertonic saline and mannitol?}

Hypertonic saline has several advantages over mannitol as a hyperosmotic agent. This can be understood by reviewing the mechanism of action of these two agents. Mannitol works by inducing diuresis, and thus increasing osmolarity through dehydration. Conversely, hypertonic saline increases the serum osmolarity directly rather than through diuresis. This difference in the mechanism of action accounts for the adverse effects that we see with the use of mannitol but not hypertonic saline.

Mannitol can cause hypotension secondary to dehydration, which could increase the morbidity of a patient with a head injury. Mannitol can also cause acute renal failure due to a combination of intravascular volume depletion and intrarenal vasoconstriction. Mannitol has been associated with rebound increase in intracranial pressure. None of these side effects are seen with the use of hypertonic saline. Hypernatremia is the most commonly reported adverse effect of hypertonic saline.

\section{Mannitol}

Increases osmolarity via diuresis

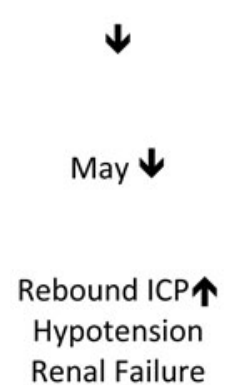

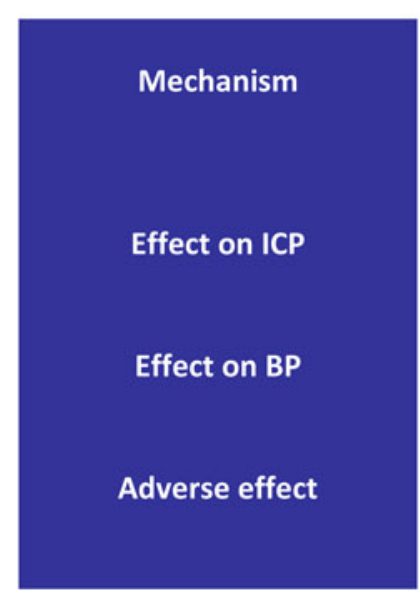

\section{Hypertonic Saline}

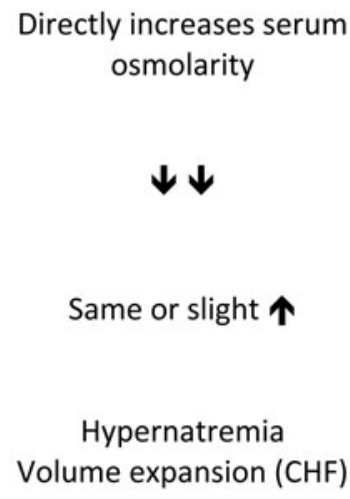




\section{What do the current guidelines recommend?}

The current (2016) Brain Trauma Foundation guidelines for the management of severe traumatic brain injury (TBI) in adults ${ }^{1}$ do not recommend the use of a specific hyperosmotic agent. The 2019 Brain Trauma Foundation guidelines for TBI in pediatrics ${ }^{2}$ recommend hypertonic saline as the only agent for intracranial pressure management. Despite the lack of guideline endorsement in the adult population, hypertonic saline has become the mainstay of treatment in the neurosurgical literature because, in addition to a more favourable side effect profile, many studies have shown it to more effectively lower intracranial pressure, ${ }^{3,4}$ with lower incidence of treatment failure ${ }^{5}$ when compared with mannitol. In the ED patient with suspected raised intracranial pressure, hypertonic saline is a reasonable, if not better, choice than mannitol.

\section{CASE RESOLUTION}

The patient was examined for signs of venous obstruction of the neck veins, and none were found. She was positioned in reverse Trendelenburg, as her injuries precluded elevating the head of the bed. A bolus of fentanyl $50 \mu \mathrm{g}$ was given, and a fentanyl infusion started at $200 \mu \mathrm{g} /$ hour. She was then paralyzed with a 50-mg bolus of rocuronium. Despite these measures her right pupil remained dilated. The emergency physician gave the patient $150 \mathrm{~mL}$ of $3 \%$ hypertonic saline, as she was concerned about the potential adverse effect of hypotension from mannitol. The patient was given two more boluses of hypertonic saline during transport to the tertiary center, where she ultimately underwent decompressive craniectomy for refractory elevated intracranial pressure.

Keywords: Critical care, neurology, emergency medicine

\section{REFERENCES}

1. Carney N, Totten AM, O'Reilly C, et al. Guidelines for the management of severe traumatic brain injury. 4th ed. Neurosurgery 2016;0:1-10.

2. Kochanek PM, et al. Guidelines for the management of severe traumatic brain injury, 3rd edition: update of the brain trauma foundation guidelines, executive summary. Pediatr Crit Care Med 2019;20(3):280-9.

3. Kamel H, et al. Hypertonic saline versus mannitol for the treatment of raised intracranial pressure: a meta-analysis of randomized clinical trials. Crit Care Med 2011;39(3);554-9.

4. Ali A, et al. Comparison of $3 \%$ hypertonic saline and $20 \%$ mannitol for reducing intracranial pressure in patients undergoing supratentorial brain surgery: a randomized, double-blind clinical trial. 7 Neurosurg Anesthesiol 2018;30(2):171-8.

5. Burgess $\mathrm{S}$, et al. A systematic review of randomized controlled trials comparing hypertonic saline solutions and mannitol for traumatic brain injury: implications for emergency department management. Ann Pharmacother 2016;50(4):291-300. 